\title{
Vivienda social en Guadalajara ¿una estrategia viable para atenuar la expansión urbana metropolitana?
}

\author{
Eugenio Arriaga Cordero ${ }^{1}$, Paola Romero Gutiérrez ${ }^{2}$ \\ Centro de Estudios Urbanos y de Vivienda, \\ ESARQ - Escuela Superior de Arquitectura y Artes. Guadalajara, México \\ E-mail: 1earriaga@esarq.edu.mx, 2promero@esarq.edu.mx
}

\begin{abstract}
Resumen. La población urbana en México se duplicó entre 1980 y 2010, mientras que la extensión territorial (urban sprawl) de las ciudades se incrementó en promedio 10 veces (IMEPLAN, 2016). Este crecimiento poco sustentable se replica en el Área Metropolitana de Guadalajara (AMG), donde la población también se duplicó pero la extensión de su territorio se triplico. El crecimiento del territorio supone la generación de problemas medio ambientales, sociales, y económicos, al incrementar la dependencia del automóvil privado para funcionar eficientemente, en un modelo de desarrollo urbano disperso, fragmentado y de baja accesibilidad, dado el aumento de las distancias entre los lugares de residencia y los servicios elementales. En contraste, el municipio de Guadalajara ha perdido población en las últimas décadas (Inegi, 2015). Lo anterior contribuyó a una pérdida del 18\% de su densidad poblacional. Mientras que la periferia metropolitana, por ejemplo, el municipio de Tlajomulco de Zúniga, aumentó su población en el mismo periodo de tiempo. Entre las razones que explican la migración intra-municipal de Guadalajara a la periferia metropolitana, está el aumento del costo de la vivienda en Guadalajara (Sociedad Hipotecaria Federal, 2019) combinado con la baja del ingreso promedio de sus habitantes (Observatorio de Salarios, 2016). Y por otro lado, el aumento en la oferta de vivienda social en el municipio de Tlajomulco de Zúniiga. El presente artículo propone explorar una serie de políticas de vivienda social para que grupos de bajos ingresos accedan a vivienda asequible en la ciudad consolidada, con el propósito de evitar que el crecimiento del territorio del AMG continúe. Entre las alternativas de política de vivienda que se exploran destacan la vivienda en alquiler, la vivienda inclusiva, los fideicomisos/bancos de tierra y el derecho de tanteo y retracto.
\end{abstract}

Palabras clave: Sustentabilidad, vivienda social, expansión

urbana.

\section{Introducción}

En la actualidad existe una legítima preocupación sobre grandes problemas medioambientales asociados con el fenómeno del cambio climático, en parte agravados por la sobre dependencia del automóvil privado en un contexto de expansión urbana (urban sprawl). No obstante se ha posicionado en la agenda pública la urgente necesidad de disminuir las emisiones contaminantes, para ello sería necesario reducir el uso del automóvil, lo que resulta poco factible dado que éste modo de transporte está irremediablemente vinculado al diseño de nuestras ciudades. En este contexto cobra importancia la forma urbana de nuestras ciudades. El AMG ha seguido un modelo basado en la expansión urbana que produce ciudades dispersas, fragmentadas $\mathrm{y}$ con baja accesibilidad. Además, el modelo de expansión urbana implica la transferencia de suelo agrícola, bosques y zonas ecológicamente 
frágiles, a áreas dedicadas a usos urbanos como la vivienda, lo que afecta negativamente la calidad del agua, el suelo y la biodiversidad (Anderson et al., 1996).

Adicionalmente, el modelo de expansión urbana ha exacerbado la segregación residencial de los grupos más vulnerables en la periferia metropolitana de Guadalajara. Lo anterior es resultado de lo que la CEPAL (2016) llama "heterogeneidad territorial", Soja (2010) "desigualdad distribucional" y Harvey (2010) define como "injusticia territorial". Estos conceptos describen esencialmente como los residentes de municipios consolidados, en comparación con los municipios de la periferia, gozan de más y mejores servicios públicos y oportunidades de bienestar material. En contraste, los municipios que conforman la periferia metropolitana se caracterizados por altos niveles de pobreza, baja calidad y menor cobertura en los servicios públicos; mayor exposición a riesgos de contaminación e inseguridad; y más largas distancias que deben recorrerse para acceder a servicios, empleo y actividades de entretenimiento, produciendo altos niveles de exclusión social (Arriaga, 2019).

Las ciudades mexicanas han experimentado el agravamiento del problema de la expansión urbana en las últimas décadas. La población urbana en México se duplicó entre 1980 y 2010, mientras que la expansión urbana de las ciudades se incrementó en promedio 10 veces (IMEPLAN, 2016). Lo anterior sucede también en Guadalajara donde la expansión urbana del AMG se triplico entre 1980 y 2015, mientras que la población solo se duplico (Ver Tabla 1). Cabe destacar que el IMEPLAN (2016) estima un escenario tendencial de crecimiento de suelo urbano para el período 2015-2045, de $57 \%$. Lo que sin duda aumentará la magnitud de las externalidades negativas.
De acuerdo con Ewing et al., (2003) la expansión urbana se caracteriza por producir desarrollos residenciales dispersos, desconectados y de baja densidad; bajo una rígida separación de usos de suelo, donde las residencias están aisladas de los sitios de consumo, empleo y entretenimiento. Por su parte Anderson et al (1996) agrega que la expansión urbana implica un crecimiento de los límites metropolitanos hacia el exterior. Ésta supone una reducción en la intensidad de todas las formas de uso de suelo, medido como densidad poblacional y de negocios. El modelo de ciudad "compacta" es la antítesis del modelo de "expansión urbana".

La adopción del modelo de expansión urbana en México tiene múltiples causas. Entre ellas destaca la construcción de infraestructura vial para facilitar el uso del automóvil y el amplio acceso a crédito para la compra de vivienda social en lugares remotos de la periferia metropolitana, donde se construyeron desarrollos residenciales sobre tierra barata, sin importar que no tuvieran acceso a servicios públicos, ni equipamiento urbano. $\mathrm{La}$ característica principal de la expansión urbana, por tanto, es una muy baja accesibilidad. Es decir, en la periferia metropolitana es difícil encontrar servicios suficientemente cerca como para acceder a éstos caminando. Esto vuelve indispensable el uso del automóvil para funcionar eficientemente (Giuliano, 2005). Por lo anterior, se antoja difícil reducir el uso del automóvil $\mathrm{y}$, por tanto, de las externalidades negativas que provoca en los ámbitos medio ambientales, sociales y económicos, entre las que destacan las siguientes:

\section{Externalidades medio-ambientales}

El aumento del tráfico en Guadalajara ha contribuido a que baje la velocidad promedio

\begin{tabular}{|l|l|l|}
\multirow{2}{*}{ Variable } & \multicolumn{2}{|l|}{ Área Metropolitana } \\
\cline { 2 - 3 } & 1980 & 2015 \\
\hline Expansión del territorio & 22,329 ha & 69,250 ha \\
\hline Población & 2 '371,278 & 4'865,122 \\
\hline
\end{tabular}

Fuente: IMEPLAN, 2016.

Tab.1. Crecimiento de la población y el territorio urbanizado en el AMG, 1980 y 2015. 
del automóvil $(23.5 \mathrm{~km} / \mathrm{hr})$ y aumente el promedio de tiempo por viaje (70 minutos en Guadalajara, y dos horas en Tlajomulco de Zúñiga) (Jalisco Cómo Vamos, 2019). Por su parte la expansión urbana ha contribuido al aumento de la distancia promedio de los viajes. Lo anterior ha explica por qué los sectores del transporte y la construcción producen en conjunto cerca del $35 \%$ de las emisiones de gases de efecto invernadero (INECC, 2018). Es decir, la construcción masiva de fraccionamientos de interés social en áreas cada vez más remotas de la periferia, junto con la pavimentación de calles y traslados más largos para acceder a estos puntos, tienen consecuencias ambientales importantes, como la afectación de la recarga de los mantos freáticos, contribuye a provocar inundaciones y eleva la temperatura al generar “islas de calor" (Sánchez, 2014).

\section{Externalidades sociales}

El $92 \%$ de la población mundial respira un aire muy contaminado (OMS, 2019). Cada año mueren 3 millones de personas en el mundo por exposición a contaminantes en el aire (ibid). Riesgo que corren principalmente los bebés y niños: 570,000 menores de cinco años fallecen como consecuencia de infecciones respiratorias. También aumenta el riesgo de sufrir cardiopatías, accidentes cerebrovasculares y cáncer a lo largo del ciclo de vida (OMS, 2018). En México hay $20 \mathrm{mil}$ muertes anuales debido a la contaminación del aire, principalmente entre mayores de 65 años (ENSANUT, 2016). Durante 2011 en el AMG el 39\% de los vehículos tenían más de 10 años de antigüedad, los cuales contaminan $400 \%$ más que los modelos más recientes (INECC, 2011).

Las lesiones producto de incidentes viales son la causa principal de muerte entre adolescentes en el mundo: En 2015 causaron la muerte de 115,000 , la mayoría peatones, ciclistas y motociclistas (OMS, 2017). En México, los incidentes de tráfico provocan 24 mil muertes, 40 mil discapacitados y 750 mil heridos al año (SEDATU, 2014). Jalisco es el segundo estado con mayor número de fallecidos en incidentes viales, después de la
Ciudad de México (Secretaría de Salud, 2016). Se puede decir que la forma urbana afecta la proclividad de hacer ejercicio. Diversos estudios han encontrado que las personas que viven en comunidades compactas (densas, conectadas, y de usos mixtos), tienden a hacer más actividad física que quienes viven en zonas suburbanas. Lo anterior es importante porque la inactividad física contribuye a elevar el riesgo de enfermedades crónicas y condiciones como el sobre peso y la obesidad, hipertensión, diabetes, cáncer, enfermedades cardiacas, entre otras, que cuestan mucho dinero al sector salud. El estilo de vida sedentario y de poca actividad física, en parte producto de la alta tasa de motorización del país, ha provocado que la obesidad en las ciudades mexicanas sea de $33 \%$ y el sobrepeso $39 \%$, lo que contribuye a enfermedades crónicas como la diabetes, que además son muy costosas (OECD, 2017). En Guadalajara, la contaminación auditiva también provoca severas consecuencias, como la pérdida o disminución de la capacidad auditiva, enfermedades fisiológicas y psicológicas que afectan el equilibrio, el sistema nervioso, el sueño, y el rendimiento laboral (Iteso, 2002).

Externalidades económicas

El costo promedio anual por el diagnóstico y tratamiento de la diabetes mellitus en 2008 fue de 77 mil pesos y de 70 mil pesos por enfermedades cardiovasculares (Gutiérrez et al., 2013). En la ZMG durante el año 2009, la contaminación atmosférica costó más de 4,000 millones de pesos; los incidentes de tráfico, 4,970 millones; la congestión vial, 10,635 millones; el ruido, 1,615 millones; (SEDATU, 2014; ITDP, 2012). Estos datos confirman lo pernicioso de las externalidades negativas asociadas al uso del automóvil.

\section{Perdida de población en Guadalajara y migración intra-municipal}

El caso del municipio de Guadalajara no se ajusta al patrón de expansión urbana y población metropolitano. Guadalajara ha perdido cerca de 200,000 habitantes en los últimos años, al pasar de 1'650 mil habitantes en 1990 a 1,460 mil en el año 2015 (Inegi, 2015). En contraste, la periferia metropolitana aumentó su población en el mismo periodo de tiempo. La migración 
intra-municipal del centro hacia la periferia metropolitana se explica, en parte, por (i) el aumento del costo de la tierra y la vivienda en Guadalajara (Sociedad Hipotecaria Federal, 2019), (ii) el bajo ingreso de la población en el AMG: $69 \%$ de la población empleada gana alrededor de 7 mil 300 pesos mensuales y solamente un $8 \%$ tiene un ingreso superior a los 5 VSM (Observatorio de Salarios, 2016, ENOE, 2017)., y (iii) el aumento en la oferta de vivienda social en la periferia metropolitana.

Este es el contexto en el que se da la expansión urbana del AMG, con las externalidades negativas producto del uso del automóvil. A continuación se discute una serie de políticas de vivienda que pueden contribuir a que grupos de bajos ingresos accedan a vivienda asequible en la ciudad consolidada de Guadalajara, y, por tanto, atenuar la expansión urbana. Las alternativas de política son la vivienda en alquiler, vivienda inclusiva, fideicomisos/ bancos de tierra, y el derecho de preferencia urbana "tanteo y retracto".

\section{Políticas sociales de vivienda Vivienda social en alquiler}

La vivienda en alquiler alcanza niveles muy altos en países como Alemania y Austria: 60\% y $50 \%$, respectivamente. El caso de la ciudad de Berlín es paradigmático: 89\% (UN-Hábitat, 2003). La vivienda en alquiler en México ha venido creciendo recientemente: prácticamente se duplicó entre los años 2000 y 2010, al pasar de 2,8 a 4 millones de unidades. Guadalajara es una de las ciudades donde más ha crecido: el 33\% de la vivienda es en renta (Inegi, 2000, 2010).

La vivienda en alquiler es una alternativa para que grupos de bajos ingresos con limitado acceso a vivienda propia en la "ciudad consolidada", acceda a ella por medio de una renta más baja de lo que ofrece el mercado por una vivienda similar en la misma zona. Los programas de vivienda en alquiler funcionan por medio de (i) subsidios a la oferta: incentivos fiscales y financiamiento preferencial a desarrolladores privados para la construcción de vivienda en alquiler; y (ii) a la demanda: apoyo a personas en desventaja social (desempleo, bajo ingreso, tipo y tamaño de familia, edad o discapacidad) que requieren ayuda para el pago del alquiler.

El incremento del costo de la vivienda en las ciudades, los cambios sociales y demográficos (Ponce, 2015) son condiciones que han contribuido al crecimiento de la vivienda en renta. Entre las ventajas de la vivienda en renta destaca su contribución a la renovación de los centros urbanos; el acceso a hogares para grupos en desventaja social; la flexibilidad que ofrece a trabajadores estacionales; el incremento a empleo y servicios (contribuyendo a la reducción de la exclusión social a la que se ven expuestos los grupos de bajos ingresos desplazados a la periferia metropolitana); y el incremento a la mezcla de estratos socioeconómicos.

\section{Bancos de terrenos y fideicomisos de tierra comunitaria}

Los Bancos de Tierra surgen en la década de 1960 para facilitar a los gobiernos adquirir propiedades excedentes, con frecuencia, para incrementar las unidades de vivienda social, de propiedad y renta (Alexander, 2008). Esta política florece en un contexto en el que el mercado de bienes raíces no funciona eficientemente al mantener los precios de la tierra muy altos (ibid).

Los fideicomisos comunitarios adquieren la tierra en las que unidades de vivienda se alquilan durante 99 años a cooperativas que proporciona viviendas para familias de ingresos bajos, quienes adquieren todos los derechos de un propietario de vivienda, excepto el derecho a venderla con ganancia. Su precio de venta se establece mediante una fórmula aprobada por el fideicomiso, que generalmente permite recuperar el precio de compra, más las mejoras a la vivienda, y algún ajuste por costo de vida, pero excluyendo el valor de la tierra, que permanece en el fideicomiso (Alexander, 2008). Este enfoque puede contribuir al aumento de la oferta de vivienda asequible para familias de bajos ingresos. El fideicomiso establece reglas básicas para garantizar que la vivienda esté disponible permanentemente para las familias que la necesitan, a los precios de alquiler más asequibles posibles.

De acuerdo con Madden y Marcuse (2016) 
entre las ventajas de estos fideicomisos destaca que contribuyen a controlar y mantener el precio de la renta bajo, cuando la tierra adquirida por el gobierno no es vendida, sino rentada. Permiten la creación de viviendas asequibles de manera permanente, para los hogares de bajos ingresos, e incluso para residentes futuros. Reducen la intensidad de la desigualdad inducida por un mercado privado que considera a la vivienda por su valor de cambio y no por su valor de uso. También contribuyen a desarrollar comunidades y estabilizan vecindarios (Alexander, 2008). Por otro lado, también pueden generar conflictos si la compensación ofrecida por el gobierno a los dueños de la tierra, es considerada poca, lo que no asegura su estabilidad.

De cualquier forma, estos enfoques eliminan la variable del costo de la tierra, facilitando la posibilidad de ofrecer vivienda asequible para grupos de bajos ingresos, lo que evita su desplazamiento a la periferia. Lo anterior es posible, en parte, porque en ambos casos se renuncia a la posibilidad de especular con un aumento anormal en el valor de la tierra y la vivienda.

\section{Vivienda inclusiva (Inclusionary Zoning)}

Esta política busca combinar familias de bajos y altos ingresos en un mismo desarrollo (Madden y Marcuse, 2016; Schuetz, et al., 2009). Regularmente funciona mediante un intercambio de unidades de vivienda para grupos en pobreza a cambio de permiso a los desarrolladores privados para construir más pisos en un edifico. Los programas de vivienda inclusiva son políticas locales que aprovechan los beneficios económicos del aumento del valor de los bienes raíces para crear viviendas asequibles. Con frecuencia estos programas requieren que los desarrolladores vendan o alquilen del 10 al 30 por ciento de las nuevas unidades residenciales a personas de bajos ingresos (Madden y Marcuse, 2016).

Hay distintos tipos de esta política en términos de plazos, tipo de apoyo, y porcentaje de vivienda. En algunas ciudades es una política obligatoria mientras que en otras es voluntaria (Schuetz et al., 2009). La idea central es que las autoridades locales reciben facultades adicionales para garantizar que nuevos desarrollos de vivienda incluyan una proporción de viviendas asequibles para cubrir una demanda creciente de vivienda para grupos en desventaja social (Varady y Walker, 2003). Esta política permite nuevos desarrollos privados de vivienda más densos y altos en áreas establecidas para ello.

Esencialmente las unidades reservadas para grupos de bajos ingresos son pagadas con las utilidades generadas por la vivienda excedente autorizada, a precio de mercado. Las preguntas clave de esta política, de acuerdo con Maden y Marcuse (2016) son: ¿quién se incluye en el programa y en qué proporción? Es decir, es un tema de límites de ingreso y proporción de participantes. La respuesta es entre más bajos sean los ingresos de los grupos atendidos y mayor sea la proporción requerida de éstos para ser incluidos, será mejor.

Este es un programa destinado a actuar contra la discriminación ejercida por el mercado de la vivienda, al asegurar que personas en desventaja social accedan a una vivienda digna en zonas centrales de la ciudad. Por tanto, este programa busca disminuir la segregación residencial, una forma de discriminación que restringe oportunidades de acceso a empleo y servicios de calidad, e inhibe la diversidad social.

En Nueva York, este programas se conoce como el " $80-20 "$ ". Es decir, el $80 \%$ de las viviendas son de ingresos altos, mientras que el 20\% de ingresos medios y bajos (Schwartz y Tajbakhsh, 1997). En San Francisco y Washington D.C., las leyes estatales exigen que las ciudades den "bonos de densidad" a los desarrolladores para construir vivienda asequible (Schuetz, et al., 2009). Quienes están a favor de esta política argumentan que impulsa la vivienda social sin tener que gastar recursos públicos vía subsidios, mientras que quienes están en contra afirman que esta política eleva los precios de las viviendas y reduce su suministro (Schuetz et al., 2009). En California, de 1988 a 2005, la política incrementó las viviendas multifamiliares y redujo las unifamiliares (Bento et al., 2009). 


\section{Derecho de preferencia urbana "tanteo y retracto"}

Una alternativa que tienen los gobiernos locales para controlar el costo del suelo y la vivienda y la especulación inmobiliaria, es la utilizada en las ciudades de Barcelona y Paris: el derecho de "tanteo y retracto" (Ampudia, 2017). Este instrumento faculta a las ciudades a ofrecer comprar tierra y vivienda en el mercado privado en áreas previamente seleccionadas, a un precio regulado, para incrementar las unidades de vivienda social.

Por lo regular la adquisición de la vivienda se da por medio de la integración de un fondo público para ese efecto. Ampudia (2017) apunta que el Ayuntamiento de Barcelona ha adquirió tierra y vivienda por este medio en barrios centrales, a precio por debajo del mercado, para desarrollar vivienda pública o brindar espacios para actividades económicas de interés social, protegiendo a los comercios que están siendo expulsados por el fenómeno de la gentrificación. El Ayuntamiento de Barcelona en algunos casos utiliza un modelo de cesión de uso de la tierra o la vivienda por 75 años a cooperativas, para administrar la vivienda social.

El Ayuntamiento de Paris cuenta con el derecho de preferencia urbana, mediante el cual puede adquirir suelo e inmuebles en venta en el mercado privado en "polígonos de actuación" definidos para ello, por medio del instrumento de tanteo y retracto. Al igual que Barcelona, lo hace a precios por debajo del mercado. Esta ha resultado una estrategia útil para atemperar la gentrificación.

El proceso de compra funciona de la siguiente manera: el Ayuntamiento tiene el derecho de ser el primero en hacer una oferta de compra con precio regulado de cualquier inmueble que salga a la venta en el mercado privado (Burón Cuadrado, 2006). El notario tiene la obligación de notificar al Ayuntamiento la intención de la venta de un inmueble, para que en un plazo menor a dos meses pueda responder si la oferta es de su interés o no. De tal manera que si el inmueble es del interés del municipio, menciona Ampudia (2017) se procederá a determinar el precio, el cual, puede variar a la baja de lo establecido por el propietario. Si se está de acuerdo en el nuevo precio, se procede a la compra. De lo contrario, el propietario puede ya sea solicitar a un juez determine un nuevo precio o abandonar la transacción.

\section{Conclusiones}

La expansión urbana del AMG ha derivado en un modelo de expansión urbana dañino que depende de manera desmesurada del uso del automóvil privado. El costo de las externalidades negativas derivadas del uso del auto en materia medio ambiental, social y económica son muy altas. Una de las causas principales de la expansión urbana $\mathrm{y}$, por tanto, de la migración intra-municipal de los habitantes más pobres hacia la periferia metropolitana, es (i) el alto costo de la tierra y la vivienda en la ciudad de Guadalajara, (ii) los bajos salarios de sus habitantes y (iii) el crecimiento desmesurado de la oferta de vivienda social en la remota periferia urbana.

Las políticas sociales de vivienda discutidas en este artículo pueden contribuir a despejar de la ecuación de la vivienda social intraurbana el alto costo del suelo en Guadalajara. $\mathrm{Y}$, por tanto, ofrecer vivienda asequible para la población en desventaja social, evitando su desplazamiento involuntario hacia la periferia metropolitana, lo que incrementa la expansión del territorio metropolitano.

Por tanto, diseñar e implementar una política social alrededor de la vivienda que ofrezca la posibilidad a los grupos más pobres de vivir en la ciudad "compacta" y, por tanto, beneficiarse de accesibilidad a servicios públicos de calidad, equipamiento urbano, y más y mejores oportunidades de empleo puede ser considerada una de las más altas prioridades de los gobiernos locales en México.

Ahora bien, como consecuencia de la expansión urbana del AMG el proceso de diseño de las políticas públicas se da en un contexto de fragmentación territorial, donde múltiples actores atienden una parte pequeña del total del territorio (OECD, 2015). Por tanto, es de vital importancia asegurar una coordinación eficiente entre las áreas de política de transporte, uso de suelo y vivienda para producir ciudades más sustentables y 
compactas, donde el problema de la expansión urbana pueda sea atemperado.

Además, es necesario incrementar todavía la accesibilidad en el municipio de Guadalajara, por medio de una mayor inversión en transporte público masivo, que drive en un servicio frecuente, seguro y de alta calidad; elevar la densidad poblacional y de negocios en sus barrios, y la mezcla de usos de suelo (Boarnet y Crane, 2001; Handy et al., 2005); al tiempo de incrementar la oferta de vivienda asequible en Guadalajara, asignatura pendiente en este municipio. Sin estas condiciones, seguiremos sufriendo irremediablemente las externalidades negativas producto del patrón de expansión urbana seguido al pie de la letra.

\section{Referencias}

Alexander, F. 2008. Land Banking as Metropolitan Policy. Blueprint for American Prosperity, Metropolitan Policy Program at Brookings.

Anderson, W. P., Kanaroglou, P. S., \& Miller, E. J. 1996. "Urban form, energy and the environment: a review of issues, evidence and policy". Urban studies, 33(1), 7-35.

Ampudia Farias, A. 2017. Políticas, instrumentos de gestión y ampliación del parque de vivienda social: del contexto europeo a estudios de caso Barcelona, España y Guadalajara, México.

Arriaga, E. 2019. "No somos iguales: Exclusión social y movilidad en el Área Metropolitana de Guadalajara: desigualdad territorial, de ingreso y género, en el viaje al trabajo". Retos vigentes de la gestión metropolitana. En impresión.

Boarnet, Marlon y Crane, Randall. 2001. Travel by Design: The Influence of urban Form on Travel. Oxford University Press: New York.

Burón Cuadrado, J. 2006. "Las reservas de suelo para la vivienda protegida: Lecciones del caso de Victoria-Gasteiz". Architecture, City and Environment. (1:2), 85-103.

CEPAL (Comisión Económica para América Latina y el Caribe). 2016. La Matriz de Desigualdad Social en América Latina. LC/G.2690. Santiago.

Encuesta Nacional de Ocupación y Empleo (ENOE). 2017. INEGI.
Encuesta Nacional de Salud y Nutrición de Medio Camino (ENSANUT). 2016. Informe final de resultados. Instituto Nacional de Salud Pública.

Ewing, R., Schmid, T., Killingsworth, R., Zlot, A., \& Raudenbush, S. 2003. "Relationship between urban sprawl and physical activity, obesity, and morbidity". American journal of health promotion, 18(1), 47-57.

Giuliano, G. 2005. "Low income, public transit, and mobility". Transportation Research Record 1927. (1), 63-70.

Gutiérrez, Cristina, Verónica Guajardo y Fernando Álvarez. 2013. "Costo de la obesidad: las fallas del mercado y las políticas públicas de prevención y control de la obesidad en México". Obesidad en México: recomendaciones para una política de Estado. UNAM: México, 348-359.

Handy, Susan; Cao, Xinyu; and Mokhtarian, Patricia. 2005. "Correlation or causality between the built environment and travel behavior? Evidence from Northern California". Transportation Research Part D. 10: 427-444.

Harvey, D. 2010. The Enigma of Capital and the Crisis this Time, Londres: Profile.

Instituto Nacional de Ecología y Cambio Climático (INECC). 2011. Estudio de emisiones y actividad vehicular en la zona metropolitana de Guadalajara, Jalisco.

2018. Presentación del Inventario de Emisiones de Gases y Compuestos de Efecto Invernadero 1990-2015.

INEGI. 2010. Censo de Población y Vivienda. 2010. Censo de Población y Vivienda.

2015. Encuesta Intercensal.

2017. Accidentes de tránsito terrestre en zonas urbanas y suburbanas. Rescatado de: https:// www.inegi.org.mx/sistemas

Instituto Metropolitano de Planeación del Área Metropolitana de Guadalajara (IMEPLAN). 2016. PotMet. Plan de Ordenamiento Territorial Metropolitano del AMG.

ITDP. 2012. Transformando la movilidad urbana en México, Instituto de Políticas para el Transporte y Desarrollo México, Embajada Británica en México.

ITESO, Ceit y SVyT. 2002. Movilidad. Una visión estratégica en la Zona Metropolitana de Guadalajara, México. 
Jalisco Cómo Vamos. 2012. Así vamos en Jalisco. Reporte de indicadores sobre calidad de vida 2012.

Madden, D., \& Marcuse, P. 2016. In defense of housing. The politics of crisis.

Observatorio de Salarios, Ibero Puebla. 2016. Informe Anual del Observatorio de Salarios 2016. Los salarios y la desigualdad en México, recuperado el 25 de octubre de 2019: http://redsalarios.org/informes/leer/ informe_2016_observatorio_de_salarios/56

OMS. 2017. ¡No contamines mi futuro! El impacto de los factores medioambientales en la salud infantil.

OECD. 2017. Panorama de la Salud 2017.

OECD. (2015). Governing the city. Paris. OECD Publishing.

OMS. 2018. ¿La herencia de un mundo sostenible? Atlas sobre salud infantil y medio ambiente.

2019. Mapa de calidad de aire en tiempo real. Rescatado el 11 de julio de 2019 de: https:// aqicn.org/map/world/es/

Sánchez, A. 2014. Las Villas Panamericanas son un crimen ecológico anunciado. Rescatado de: http://cucea.udg.mx/es/ noticia/03-dec-2014

Schuetz, J., Meltzer, R. y Been, V. 2009. “31 Flavors of Inclusionary Zoning: Comparing Policies From San Francisco, Washington, DC, and Suburban Boston". Journal of the American Planning Association. (75:4), 441-456.

Secretaría de Salud. 2016. Perfil Estatal y Perfil Nacional de Seguridad Vial.

SEDATU. 2014. Estrategia Nacional de Movilidad Sustentable, Subsecretaría de Desarrollo Urbano y Vivienda, Gobierno de México.

Sociedad Hipotecaria Federal. 2018. Índice SHF de Tercer Trimestre de Precios de la Vivienda en México 2018, rescatado el 3 de mayo de 2019, de: https://www.gob.mx/shf/ documentos/indice-shf-de-precios-de-lavivienda-en-mexico-2018.

Soja, E. 2010. Seeking spatial justice. University of Minnesota Press.

UN-Hábitat. 2003. Rental Housing An essential option for the urban poor in developing countries. Nairobi: UN-Hábitat. 